\title{
Scheme-invariant NNLO evolution for unpolarized DIS structure functions
}

\author{
J. Blümlein ${ }^{\mathrm{a}}$ and A. Guffanti ${ }^{\mathrm{a}}$ \\ aDESY, Platanenallee 6, D-15738 Zeuthen, Germany
}

We discuss the combination of NNLO standard QCD evolution and scheme-invariant analysis for unpolarized DIS structure functions data as a method to reduce the theoretical errors on the determination of $\alpha_{s}\left(M_{Z}^{2}\right)$ to $\sim 1 \%$ in order to match the accuracy forseen for experimental errors from future high statistics measurements.

\section{INTRODUCTION}

The final HERA-II data on unpolarized deeply inelastic scattering (DIS) structure functions, combined with the present world data, will allow to reduce the experimental error on the strong coupling constant, $\alpha_{s}\left(M_{Z}^{2}\right)$, to the level of $1 \%$ [1]. On the theoretical side, the next-to-leading order (NLO) analyses have intrinsic limitations which allow no better than $5 \%$ accuracy in the determination of $\alpha_{s}$ 2. In order to match the expected experimental accuracy, analyses of DIS structure functions need then to be carried out at the NNLO level, which requires the knowledge of the $\beta$-function and anomalous dimensions at the 3-loop level and the massless and massive 2loop Wilson coefficients. With the recent computation of the 3-loop anomalous dimensions [3], the whole scheme independent set of quantities is known, thus allowing a complete NNLO study of DIS structure functions. At the same time we think that combining the standard QCD analysis and fits based on scheme-invariant evolution will provide a valuable tool to reduce theoretical and conceptual uncertainties in high-precision analyses aiming at $1 \%$ accuracy.

Our final goal is to perform the full NNLO analysis of DIS structure functions aiming at an highaccuracy determination of $\alpha_{s}$ and the extraction of a set of parton distribution functions (PDFs) with fully correlated errors. A complete analysis refers to both the singlet and non-singlet evolution. In the present letter we will concentrate only on the singlet sector, referring the reader to the recent non-singlet analysis [4].

\section{QCD EVOLUTION EQUATIONS}

Evolution equations of DIS structure functions depend, in the standard QCD approach, on two arbitrary scales which are introduced in the calculation: the renormalization and the factorization scales.

The renormalization scale dependence of any observable can be removed only summing the perturbative series to all orders. Its presence is then unavoidable in any fixed order result. Moreover, the dependence of the result on the variation of this unphysical scale can be used to give a rough estimate of the theoretical error due to higher order corrections.

On the other hand, if we consider the dependence of the result on the factorization scale we may follow two approaches. The first one is to consider the evolution of parton distribution functions which are process-independent but depend on the adopted factorization scheme. The second one is to study evolution equations for physical observables. In these equations the rôle of anomalous dimensions for mass factorization is played by physical anomalous dimensions, which are independent of the factorization scheme but 
depend on the process and the observables considered. These two choices define what we call the standard QCD analysis and the scheme-invariant analysis.

In the standard QCD analysis one introduces a parameterization for the different PDFs at a given reference scale. The PDFs are then evolved up to the actual scale of the process, solving the evolution equations for mass factorization. Structure functions are then constructed as a convolution of the PDFs and the corresponding Wilson coefficients. As a last step, a multi-parameter fit is then performed to extract the value of the coupling constant and determine the parameters entering the PDF parameterization.

In a scheme-invariant analysis the parameterization of the observable at the reference scale $Q_{0}^{2}$ is extracted from the data. The value of the observable at the scale $Q^{2}$ is determined solving the evolution equations with physical anomalous dimensions as evolution kernels. Finally a oneparameter fit is performed to extract the value of $\alpha_{s}\left(M_{Z}^{2}\right)$. Once the analysis is completed the parton densities in any factorization scheme can be extracted along with the respective experimental errors. The advantage of considering factorization scheme invariant evolution equations for physical observables resides in the fact that the input distributions are observables. Full exploitation of this advantage requires therefore high statistics measurements to minimize errors on the input distributions. Furthermore, the correlations between the measured input distributions have to be considered in detail.

Once more we would like to stress that the two analyses are complementary and not mutually exclusive. Thus performing both of them and comparing the extracted values of $\alpha_{s}\left(M_{Z}^{2}\right)$, or correspondingly $\Lambda_{Q C D}$, provides a test of stability to determine the QCD parameter.

\section{PHYSICAL ANOMALOUS DIMEN- SIONS}

When considering the singlet evolution the quarksinglet and gluon PDFs can be mapped into a pair of structure functions via the matrix of Wilson coefficients, $\mathbf{C}^{N},[5]$ :

$$
\left(\begin{array}{c}
F_{A}^{N} \\
F_{B}^{N}
\end{array}\right)=\left(\begin{array}{ll}
C_{A, \Sigma}^{N} & C_{A, g}^{N} \\
C_{B, \Sigma}^{N} & C_{B, g}^{N}
\end{array}\right)\left(\begin{array}{c}
\Sigma^{N} \\
G^{N}
\end{array}\right) .
$$

In Eq. (1), as we will do in the following, we work in Mellin space, where convolutions are ordinary products.

The singlet evolution equations read

$\frac{d}{d t}\left(\begin{array}{c}F_{A}^{N} \\ F_{B}^{N}\end{array}\right)=-\frac{1}{4} \mathbf{K}^{\mathbf{N}}\left(\begin{array}{c}F_{A}^{N} \\ F_{B}^{N}\end{array}\right)$,

where the evolution variable is

$t=-\frac{2}{\beta_{0}} \ln \frac{a_{s}\left(Q^{2}\right)}{a_{s}\left(Q_{0}^{2}\right)}$.

The coupling constant $a_{s}$ is related to the usual strong interactions coupling via the relation

$a_{s}\left(\mu^{2}\right)=\frac{\alpha_{s}\left(\mu^{2}\right)}{4 \pi}$

and its expansion to 3-loops reads

$$
\begin{aligned}
a_{s}\left(Q^{2}\right)=\frac{1}{\beta_{0} L} & \left\{1-\frac{\beta_{1} \ln L}{\beta_{0}^{2} L}\right. \\
& \left.+\frac{\beta_{1}^{2} \ln ^{2} L-\beta_{1}^{2} \ln L+\beta_{2} \beta_{0}-\beta_{1}^{2}}{\beta_{0}^{4} L^{2}}\right\},
\end{aligned}
$$

where

$L=\ln \frac{Q^{2}}{\Lambda_{Q C D}^{2}}$,

the $\beta$ function is defined as

$\mu^{2} \frac{d a_{s}\left(\mu^{2}\right)}{d \mu^{2}}=-\sum_{n=0}^{\infty} \beta_{n} a_{s}^{n+2}\left(\mu^{2}\right)$

and, in the case of $S U(3)_{c}$, the coefficients entering up to 3-loops are

$$
\begin{aligned}
& \beta_{0}=11-\frac{2}{3} N_{f}, \\
& \beta_{1}=102-\frac{38}{3} N_{f}, \\
& \beta_{2}=\frac{2857}{2}-\frac{5033}{18} N_{f}+\frac{325}{54} N_{f}^{2},
\end{aligned}
$$

with $N_{f}$ the number of flavors. The physical anomalous dimensions $\mathbf{K}^{N}$ can be expressed in 
terms of the anomalous dimensions and the Wilson coefficients as [5]

$$
\begin{aligned}
K_{I J}^{N} & =\left[-4 \frac{\partial C_{I, m}^{N}(t)}{\partial t}\left(C^{N}\right)_{m, J}^{-1}(t)\right. \\
& \left.-\frac{\beta_{0} a_{s}\left(Q^{2}\right)}{2 \beta\left(a_{s}\left(Q^{2}\right)\right)} C_{I, m}^{N}(t) \gamma_{m n}^{N}(t)\left(C^{N}\right)_{n, J}^{-1}(t)\right] .
\end{aligned}
$$

Here $\gamma_{m n}^{N}$ denotes the unpolarized anomalous dimensions which are related to the evolution kernels in $x$-space by

$\gamma_{m n}^{N}=-2 \int_{0}^{1} d x x^{N-1} P_{m n}(x), \quad m, n=q, g$

and $C_{I, m}^{N}$ are the Mellin transforms of the Wilson coefficients

$C_{I, m}^{N}=\int_{0}^{1} d x x^{N-1} C_{I, m}(x)$.

While the anomalous dimensions and the Wilson coefficients are, separately, factorization-scheme dependent quantities, the combinations (9) defining the physical anomalous dimensions are factorization scheme invariants, order by order in perturbation theory.

Different pairs of structure function can be taken into consideration, in particular:

- $F_{2}$ and $\partial F_{2} / \partial t[657$;

- $F_{2}$ and $F_{L}$ 85].

In the case of polarized DIS a combined $\overline{M S}$ and scheme invariant analysis was carried out to NLO for the structure functions $g_{1}$ and $\partial g_{1} / \partial t$ in $[\mathbf{7}$.

Here we present the NNLO physical anomalous dimensions for the coupled evolution of the structure functions $F_{2}$ and $\partial F_{2} / \partial t$, alongside with those at LO and NLO, cf. [5] :

LO :

$K_{22}^{N(0)}=0, \quad K_{2 d}^{N(0)}=-4$,

$K_{d 2}^{N(0)}=\frac{1}{4}\left(\gamma_{q q}^{N(0)} \gamma_{g g}^{N(0)}-\gamma_{q g}^{N(0)} \gamma_{g q}^{N(0)}\right)$,

$K_{d d}^{N(0)}=\gamma_{q q}^{N(0)}+\gamma_{g g}^{N(0)}$.
NLO :

$$
\begin{aligned}
& K_{22}^{N(1)}=K_{2 d}^{N(1)}=0 \\
& K_{d 2}^{N(1)}=\frac{1}{4}\left[\gamma_{g g}^{N(0)} \gamma_{q q}^{N(1)}+\gamma_{g g}^{N(1)} \gamma_{q q}^{N(0)}\right. \\
&\left.-\gamma_{q g}^{N(1)} \gamma_{g q}^{N(0)}-\gamma_{q g}^{N(0)} \gamma_{g q}^{N(1)}\right] \\
&-\frac{\beta_{1}}{2 \beta_{0}}\left(\gamma_{q q}^{N(0)} \gamma_{g g}^{N(0)}-\gamma_{g q}^{N(0)} \gamma_{q g}^{N(0)}\right) \\
&+\frac{\beta_{0}}{2} C_{2, q}^{N(1)}\left(\gamma_{q q}^{N(0)}+\gamma_{g g}^{N(0)}-2 \beta_{0}\right) \\
&-\frac{\beta_{0}}{2} \frac{C_{2, g}^{N(1)}}{\gamma_{q g}^{N(0)}}\left[\left(\gamma_{q q}^{N(0)}\right)^{2}-\gamma_{q q}^{N(0)} \gamma_{g g}^{N(0)}\right. \\
&\left.+2 \gamma_{q g}^{N(0)} \gamma_{g q}^{N(0)}-2 \beta_{0} \gamma_{q q}^{N(0)}\right] \\
&-\frac{\beta_{0}}{2}\left(\gamma_{q q}^{N(1)}-\frac{\gamma_{q q}^{N(0)} \gamma_{q g}^{N(1)}}{\gamma_{q g}^{N(0)}}\right) \\
&=\gamma_{q q}^{N(1)}+\gamma_{g g}^{N(1)}-\frac{\beta_{1}}{\beta_{0}}\left(\gamma_{q q}^{N(0)}+\gamma_{g g}^{N(0)}\right) \\
&-\frac{2 \beta_{0}}{\gamma_{q g}^{N(0)}}\left[C_{2, g}^{N(1)}\left(\gamma_{q q}^{N(0)}-\gamma_{g g}^{N(0)}-2 \beta_{0}\right)\right. \\
&\left.K_{d d}^{N(1)}\right]+4 \beta_{0} C_{2, q}^{N(1)}-2 \beta_{1} \\
&=
\end{aligned}
$$

\section{NNLO :}

$$
\begin{aligned}
K_{22}^{N(2)} & =K_{2 d}^{N(2)}=0 \\
K_{d 2}^{N(2)} & =\frac{1}{4}\left(\gamma_{q q}^{N(2)} \gamma_{g g}^{N(0)}+\gamma_{q q}^{N(0)} \gamma_{g g}^{N(2)}\right. \\
& -\gamma_{q g}^{N(2)} \gamma_{g q}^{N(0)}-\gamma_{q g}^{N(0)} \gamma_{g q}^{N(2)} \\
& \left.+\gamma_{q q}^{N(1)} \gamma_{g g}^{N(1)}-\gamma_{q g}^{N(1)} \gamma_{g q}^{N(1)}\right) \\
& +\frac{\beta_{0}}{2}\left[C_{2, q}^{N(1)}\left(\gamma_{q q}^{N(1)}+\gamma_{g g}^{N(1)}\right)\right. \\
& \left.-\left(C_{2, q}^{N(1)}\right)^{2}\left(\gamma_{q q}^{N(0)}+\gamma_{g g}^{N(0)}\right)-3 C_{2, g}^{N(1)} \gamma_{g q}^{N(1)}\right] \\
& -\beta_{0}\left[2 \gamma_{g q}^{N(0)}\left(C_{2, g}^{N(2)}-C_{2, g}^{N(1)} C_{2, q}^{N(1)}\right)\right. \\
& \left.-C_{2, q}^{N(2)}\left(\gamma_{q q}^{N(0)}+\gamma_{g g}^{N(0)}\right)+\gamma_{q q}^{N(2)}\right]
\end{aligned}
$$




$$
\begin{aligned}
& +\beta_{0}^{2}\left[3\left(C_{2, q}^{N(1)}\right)^{2}-4 C_{2, q}^{N(2)}\right] \\
& +\frac{\beta_{1}}{2}\left[\gamma_{q q}^{N(1)}+C_{2, g}^{N(1)} \gamma_{g q}^{N(0)}\right. \\
& \left.-C_{2, q}^{N(1)}\left(\gamma_{q q}^{N(0)}+\gamma_{g g}^{N(0)}+2 \beta_{0}\right)\right] \\
& -\frac{\beta_{1}}{2 \beta_{0}}\left(\gamma_{q q}^{N(1)} \gamma_{g g}^{N(0)}+\gamma_{q q}^{N(0)} \gamma_{g g}^{N(1)}\right. \\
& \left.-\gamma_{q g}^{N(1)} \gamma_{g q}^{N(0)}-\gamma_{q g}^{N(0)} \gamma_{g q}^{N(1)}\right) \\
& +\frac{3}{4} \frac{\beta_{1}^{2}}{\beta_{0}^{2}}\left(\gamma_{q q}^{N(0)} \gamma_{g g}^{N(0)}-\gamma_{q g}^{N(0)} \gamma_{g q}^{N(0)}\right) \\
& -\frac{\beta_{2}}{2 \beta_{0}}\left(\gamma_{q q}^{N(0)} \gamma_{g g}^{N(0)}+\gamma_{q g}^{N(0)} \gamma_{g q}^{N(0)}\right) \\
& +\frac{1}{\gamma_{q g}^{N(0)}}\left\{2 \beta_{0}^{3} C_{2, q}^{N(1)} C_{2, g}^{N(1)}\right. \\
& +\frac{\beta_{1}}{2} \gamma_{q q}^{N(0)}\left[C_{2, g}^{N(1)}\left(\gamma_{q q}^{N(0)}-\gamma_{g g}^{N(0)}\right)-\gamma_{q g}^{N(0)}\right] \\
& +\beta_{0}^{2}\left[4 \gamma_{q q}^{N(0)}\left(C_{2, g}^{N(2)}-C_{2, g}^{N(1)} C_{2, q}^{N(1)}\right)\right. \\
& -C_{2, g}^{N(1)} C_{2, q}^{N(1)}\left(\gamma_{q q}^{N(0)}-\gamma_{g g}^{N(0)}-\frac{\gamma_{q g}^{N(1)}}{C_{2, g}^{N(1)}}\right) \\
& \left.+C_{2, g}^{N(1)}\left(\gamma_{q q}^{N(1)}+C_{2, g}^{N(1)} \gamma_{g q}^{N(0)}\right)\right] \\
& +\beta_{0}\left[C_{2, g}^{N(1)} C_{2, q}^{N(1)} \gamma_{q q}^{N(0)}\left(\gamma_{q q}^{N(0)}-\gamma_{g g}^{N(0)}\right)\right. \\
& \left.+\gamma_{q q}^{N(0)}\left(C_{2, g}^{N(1)} \gamma_{g g}^{N(1)}+C_{2, g}^{N(2)} \gamma_{g g}^{N(0)}\right)\right] \\
& +\frac{\beta_{0}}{2}\left[C _ { 2 , g } ^ { N ( 1 ) } \left(\gamma_{q q}^{N(1)} \gamma_{g g}^{N(0)}+\gamma_{g q}^{N(0)} \gamma_{q g}^{N(1)}\right.\right. \\
& \left.-\frac{3}{2} \gamma_{q q}^{N(0)} \gamma_{q q}^{N(1)}\right)+\gamma_{q q}^{N(1)} \gamma_{g q}^{N(1)} \\
& \left.\left.+\left(C_{2, g}^{N(1)}\right)^{2} \gamma_{g q}^{N(0)}\left(\gamma_{g g}^{N(0)}-\frac{3}{2} \gamma_{q q}^{N(0)}\right)\right]\right\} \\
& +\frac{2 \beta_{0}}{\left(\gamma_{q g}^{N(0)}\right)^{2}}\left\{-\beta_{0}^{2}\left(C_{2, g}^{N(1)}\right)^{2} \gamma_{q q}^{N(0)}\right. \\
& +\beta_{0}\left[-C_{2, g}^{N(1)} \gamma_{q q}^{N(0)} \gamma_{q g}^{N(1)}\right. \\
& +\left(C_{2, g}^{N(1)}\right)^{2} \gamma_{q q}^{N(0)}\left(\gamma_{q q}^{N(0)}-\gamma_{g g}^{N(0)}\right. \\
& \left.\left.+\frac{\gamma_{q q}^{N(0)} \gamma_{g g}^{N(0)}}{2}\right)\right] \\
& -\frac{1}{2}\left[\left(C_{2, g}^{N(1)}\right)^{2} \gamma_{q q}^{N(0)}\left(\gamma_{q q}^{N(0)}-\gamma_{g g}^{N(0)}\right)^{2}\right. \\
& \left.-\gamma_{q q}^{N(0)}\left(\gamma_{q g}^{N(1)}\right)^{2}\right]
\end{aligned}
$$

$$
\begin{aligned}
& \left.+C_{2, g}^{N(1)} \gamma_{q g}^{N(1)} \gamma_{q q}^{N(0)}\left(\gamma_{q q}^{N(0)}-\gamma_{g g}^{N(0)}\right)\right\} \\
& K_{d d}^{N(2)}=\gamma_{q q}^{N(2)}+\gamma_{g g}^{N(2)}-4 \beta_{2} \\
& -4 \beta_{0}\left[\left(C_{2, q}^{N(1)}\right)^{2}-2 C_{2, q}^{N(2)}\right] \\
& +\left(\frac{\beta_{1}^{2}}{\beta_{0}^{2}}-\frac{\beta_{2}}{\beta_{0}}\right)\left(\gamma_{q q}^{N(0)}+\gamma_{g g}^{N(0)}\right) \\
& -\frac{\beta_{1}}{\beta_{0}}\left(\gamma_{q q}^{N(1)}+\gamma_{g g}^{N(1)}-2 \beta_{1}\right) \\
& +\frac{4 \beta_{0}}{\gamma_{q g}^{N(0)}}\left\{4 \beta_{0}\left(C_{2, g}^{N(2)}-C_{2, q}^{N(1)} C_{2, g}^{N(1)}\right)\right. \\
& +\left(\gamma_{q q}^{N(0)}-\gamma_{g g}^{N(0)}\right)\left(C_{2, g}^{N(1)} C_{2, q}^{N(1)}-C_{2, g}^{N(2)}\right) \\
& -C_{2, g}^{N(1)}\left(\gamma_{q q}^{N(1)}-\gamma_{g g}^{N(1)}-2 \beta_{1}\right) \\
& \left.-\left(C_{2, g}^{N(1)}\right)^{2} \gamma_{g q}^{N(0)}+\gamma_{q g}^{N(2)}\right\} \\
& +\frac{2 \beta_{0}}{\left(\gamma_{q g}^{N(0)}\right)^{2}}\left\{-4 \beta_{0}^{2}\left(C_{2, g}^{N(1)}\right)^{2}-\left(\gamma_{q g}^{N(1)}\right)^{2}\right. \\
& -4 \beta_{0} C_{2, g}^{N(1)}\left[\gamma_{q g}^{N(1)}-C_{2, g}^{N(1)}\left(\gamma_{q q}^{N(0)}-\gamma_{g g}^{N(0)}\right)\right] \\
& +2 C_{2, g}^{N(1)} \gamma_{q g}^{N(1)}\left(\gamma_{q q}^{N(0)}-\gamma_{g g}^{N(0)}\right) \\
& \left.-\left[C_{2, g}^{N(1)}\left(\gamma_{q q}^{N(0)}-\gamma_{g g}^{N(0)}\right)\right]^{2}\right\}
\end{aligned}
$$

The analytic structure of the physical anomalous dimensions $K_{i j}^{(n)}$ in $x$-space is difficult to obtain since they require the computation of the inverse Mellin transform of products of coefficient functions and inverse splitting functions, which are highly non-trivial already in the simplest cases (for an example see 9]). We therefore perform the evolution in Mellin space. The result, computed for integer $N$ is then analytically continued to arbitrary complex $N$ using representations of harmonic sums with arbitrary precision [10] and the $x$-space result is obtained through a single numerical contour integral.

\section{HEAVY FLAVORS CONTRIBUTION}

Heavy flavor contributions to DIS structure functions are known to be sizable in the kinematic region spanned by HERA. An example being the 


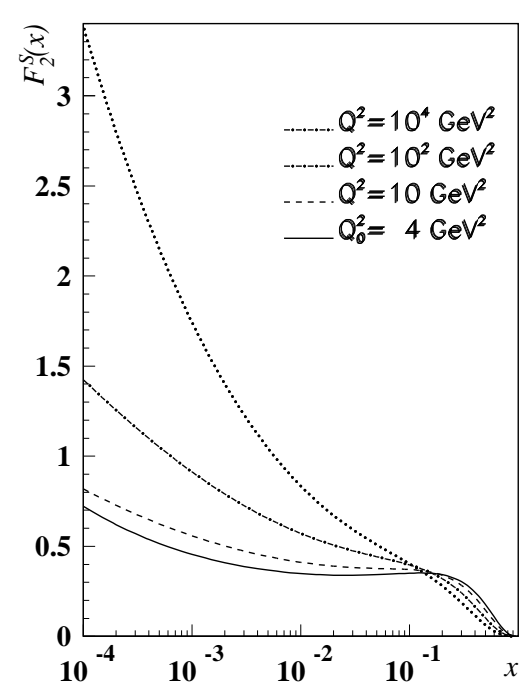

Figure 1. NLO scheme invariant evolution for the singlet part of the structure function $F_{2}$ for four light flavors.

structure function $F_{2}$ which, depending on actual event kinematics, can receive contributions from heavy flavors up to the level of $20-40 \%$. Any analysis aiming at extracting $\alpha_{s}$ form DIS structure functions data with an accuracy of $\sim 1 \%$ must, therefore, take into account heavy flavor contributions. Recently a parameterization of heavy flavor Wilson coefficients in Mellin space has been derived 11], thus allowing a direct incorporation into computer codes which solve the evolution equation in Mellin space.

\section{NUMERICAL RESULTS}

While full numerical implementation of the NNLO scheme invariant evolution is almost finished, as a preliminary result, in Fig. 1 and Fig. 2 we present the scheme invariant evolution for the structure functions $F_{2}$ and $\partial F_{2} / \partial t$ at NLO for four light flavors. In the present computation the initial form of the observables is built up as a convolution of Wilson coefficients and PDFs at the reference scale $Q_{0}^{2}=1 \mathrm{GeV}^{2}$, using parameterization of [12.

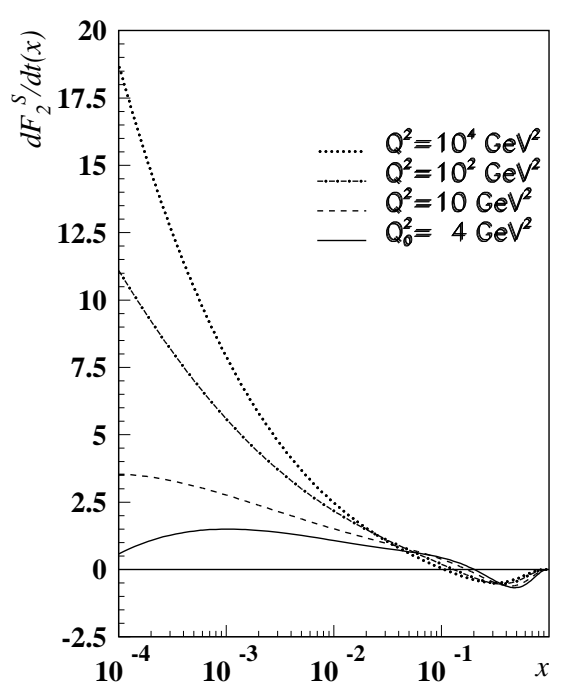

Figure 2. NLO scheme invariant evolution for the singlet part of $\partial F_{2} / \partial t$ for four light flavors.

\section{CONCLUSIONS}

The future high precision HERA-II data will allow a reduction of the experimental error on the determination of $\alpha_{s}$ to $\sim 1 \%$. On the theoretical side, the inclusion of NNLO corrections is mandatory to cope with such a level of accuracy. In view of a high accuracy determination of the strong coupling constant we think that combining the standard $\overline{M S}$ analysis and fits based on factorization-scheme invariant evolution could provide a method to have better control on theoretical and conceptual errors on $\alpha_{s}$.

Acknowledgment. This paper was supported in part by DFG Sonderforschungsbereich Transregio 9, Computergestützte Theoretische Physik.

\section{REFERENCES}

1. M. Botje, M. Klein, and C. Pascaud, hep-ph/9609489

2. J. Blümlein, S. Riemersma, W.L. van Neerven, and A. Vogt, hep-ph/9609217

3. S. Moch, J. A. S. Vermaseren and A. Vogt, Nucl. Phys. B B688 (2004) 101; B691 (2004) 129.

4. J. Blümlein, H. Böttcher and A. Guffanti, hep-ph/0407089

5. J. Blümlein, V. Ravindran and W. L. van 
Neerven, Nucl. Phys. B586 (2000) 349.

6. W. Furmanski and R. Petronzio, Z. Phys. C11 (1982) 293.

7. J. Blümlein and H. Böttcher, Nucl. Phys. B636 (2002) 225.

8. S. Catani, Z. Phys. C75 (1997) 665.

9. L. Baulieu and C. Kounnas, Nucl. Phys. B155 (1979) 429.

10. J. Blümlein and S. Kurth, Phys. Rev. D69 (1999) 014018;

J. Blümlein, Comp. Phys. Commun. 133 (2000) 76; 159 (2004) 19.

11. S. I. Alekhin and J. Blümlein, Phys. Lett. B594 (2004) 299.

12. A. D. Martin, R. G. Roberts, W. J. Stirling and R. S. Thorne, Eur. Phys. J. C 23 (2002) 73 